\title{
HYPOTHESIS
}

\section{Phenotypic modulation of chondrocytes as a potential therapeutic target in osteoarthritis: a hypothesis}

\author{
T Aigner, J Dudhia
}

One central hallmark of osteoarthritic cartilage degeneration is the loss of matrix molecules, in particular proteoglycans. However, chondrocytes of osteoarthritic cartilage are generally thought to be anabolically hyperactive. Thus, in osteoarthritic cartilage degeneration, the ongoing net loss of the cartilage matrix components is attributed not to a lack of synthesis of cartilage matrix molecules by the cartilage cells, but to an increase in matrix catabolism by most authors. ${ }^{1-3}$ In contrast, our in situ analysis on the single cell level showed a suppression of aggrecan and collagen type II expression in the chondrocytes in the upper cartilage zone, which is critical for the progression of the cartilage destruction. Phenotyping of the osteoarthritic chondrocytes in this zone furthermore shows that the decrease in anabolic activity was presumably not simply because of cell deactivation, but may entail a specific cell differentiation pathway taken by the osteoarthritic chondrocytes. Based on our in situ analyses, we suggest a three step process of cellular events as one central pathway during osteoarthritic cartilage destruction. We conclude that stimulation of matrix anabolism via redifferentiation of osteoarthritic chondrocytes may be a relevant goal in future therapeutic approaches to osteoarthritic cartilage destruction.

\section{Central observations}

The functioning of articular cartilage is dependent on its extracellular matrix, which is synthesised and maintained by the cartilage cells, the chondrocytes. The functional properties of cartilage matrix are mainly provided by a network of type II collagen fibres ${ }^{4}$ and the entrapped aggregates of the proteoglycan aggrecan. ${ }^{5}$ The hallmarks of osteoarthritic cartilage degeneration are depletion of the matrix proteoglycans, damage to the collagen network, and finally, progressing matrix erosion. ${ }^{36-8}$ Based on our own work and that of others, we present a hypothesis for the basis of progressive cartilage destruction, focusing on the selective changes that occur in the different cartilage zones.

Generally, osteoarthritic cartilage cells are thought to be anabolically hyperactive synthesising increased amounts of cartilage matrix components such as collagens and proteoglycan aggrecan. ${ }^{6-14}$ These, mostly biochemical, studies were based on an overall measurement of chondrocyte behaviour of the whole osteoarthritic cartilage and were not able to detect differences between cells of different cartilage zones. Our in situ analysis on the single cell level confirmed the overall increase in anabolic activity of the osteoarthritic chondrocytes compared with normal specimens (fig 1B, C) in terms of increased mRNA expression of the major extracellular matrix components, in particular collagen type II (fig 1G, fig 2B) and to a lesser extent aggrecan (fig 1F). ${ }^{15}$ However, the hyperactivity of matrix synthesis was restricted to the chondrocytes of the middle and deeper zones of osteoarthritic cartilage, where the extracellular matrix was histochemically still intact and no major loss of proteoglycan was detectable (fig 1E). More importantly, chondrocytes in the upper zones of osteoarthritic cartilage showed considerably less or no expression of collagen type II and aggrecan (fig $1 \mathrm{~F}, \mathrm{G}$, fig $2 \mathrm{~B}$ ). This is in agreement with a previous $\left[\mathrm{SO}_{4}\right]$ incorporation study, which also found reduced proteoglycan synthesis in the upper zones of osteoarthritic cartilage. ${ }^{17}$ The upper zone of osteoarthritic cartilage does not, of course, correspond to the superficial zone of normal articular cartilage, which is eroded in advanced disease. Thus, in the area of progressing proteoglycan loss (fig 1E) and collagen network destruction, chondrocytes are found to be in a state of hypoactivity, not hyperactivity. ${ }^{18}$ This explains, at least in part, the loss in proteoglycan content in this zone, if one assumes that the diffusion capacity of aggrecan monomers is limited and increased synthesis in one zone cannot compensate for the synthetic failure in other zones.

It is noteworthy that in many samples a transitional zone was seen, in which there was a normal pattern of proteoglycan staining in the pericellular region, but not in the interterritorial matrix (fig 1E). Whether this indicates increased proteoglycan degradation, which is no longer compensated by cells still actively 

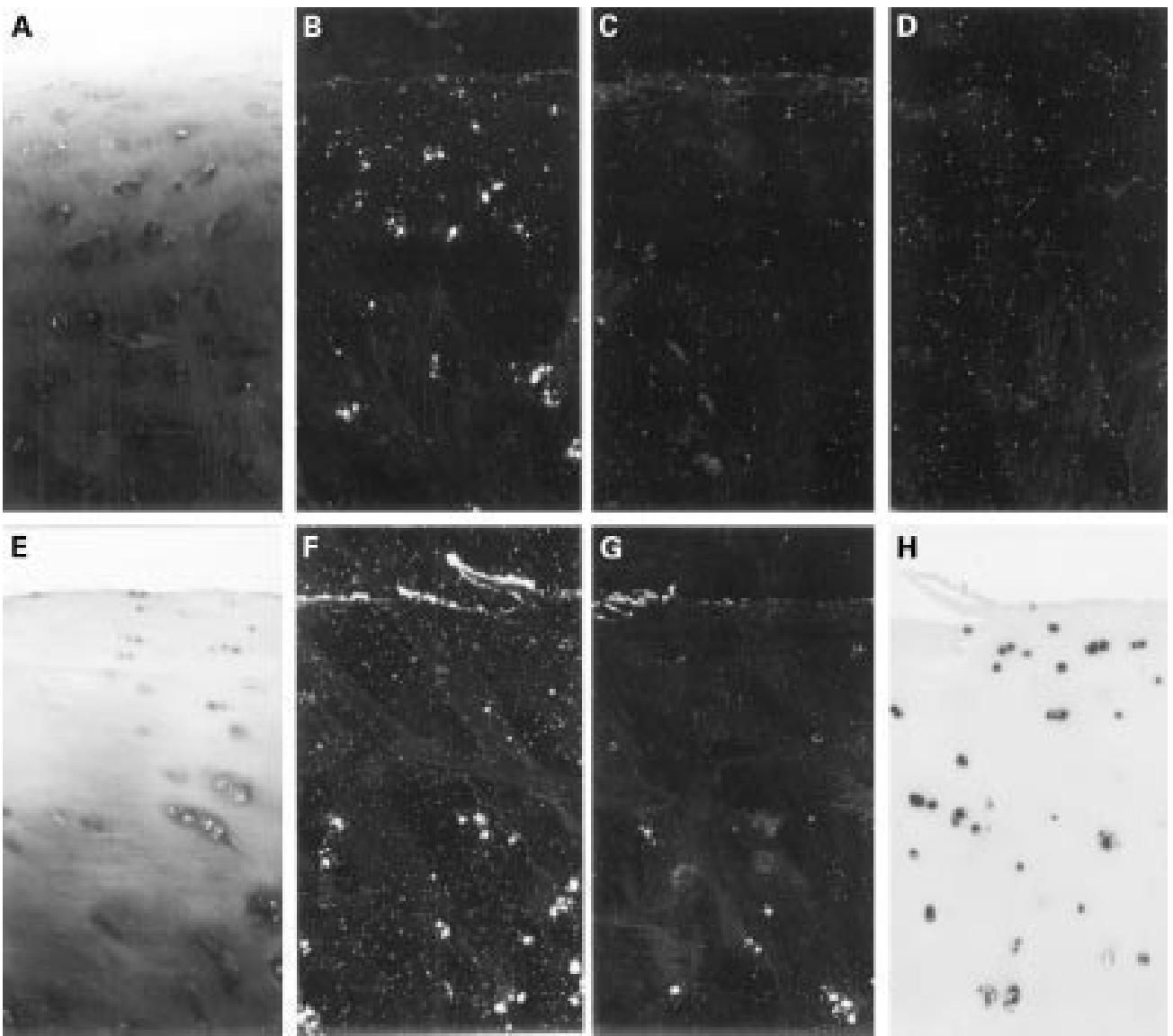

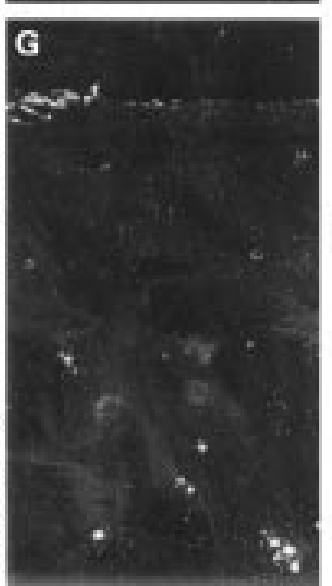

Figure 1 In situ hybridisation analysis of normal $(A-D)$ and osteoarthritic $(E-H)$ cartilage specimens using $R N A$ probes specific for the major extracellular matrix proteins of articular cartilage, aggrecan core protein (B, $F)$, and collagen type II $(C, G)$. Chondrocytes of normal articular cartilage from all specimens showed no significant expression of collagen type II $m R N A(C)$, but significant levels of aggrecan core protein mRNA in most cases (B). In contrast, analysis of osteoarthritic cartilage showed a strong activation of the expression of collagen type II $m R N A$ in the middle and upper deep zone chondrocytes in all samples $(G)$. Aggrecan $m R N A$ expression was, in comparison, much less increased in these cells $(F)$ compared with the normal specimens. Thus, $m R N A$ analysis showed osteoarthritic chondrocytes to be hyperactive in the middle and deeper zones of cartilage compared with normal specimens. Most importantly however, in the upper zone chondrocytes in most of the osteoarthritic specimens, no expression of either protein was observed (F, $G)$. Coincidentally with this area, loss of proteoglycan staining was seen $(E)$. The upper inactive chondrocytes were not necrotic as shown by strong signals for $18 S \mathrm{rRNA}(H)$. (A,E: toluidine blue; B-D,F,G: dark fields; A-D: femoral head, 68 years, Mankin's grade 1, female; E-H: femoral head, 69 years, Mankin's grade 4, female; exposure time: B-D,F: three weeks; G: four days;

$A-H$ :original magnification $\times 140$; the chosen exposure times were optimal. Longer exposures, did not increase the number of positive cells nor improve the signal to noise ratio). Methods (figs 1 and 2): 11 normal specimens from necopsies and amputations for cancer (age range 45 to 79 years) and 32 osteoarthritic cartilage slices (hip and knee replacement operations for late stage osteoarthritis; age range 52-78 years) were fixed with 4\% paraformaldehyde, decalcified, and embedded in paraffin wax. Toluidine blue and safranin $O$ stainings were performed to estimate the content of

proteoglycans. ${ }^{41}$ The samples were classified and graded according to Mankin et al. ${ }^{6}$ Specific ${ }^{35} S$-labelled RNA probes for human collagen chains $\alpha 1(I), a 1(I I), a 1(I I I), a 1(X)$, and aggrecan core protein were prepared and in situ hybridisation performed as described elsewhere. ${ }^{25}$ The riboprobe for $18 \mathrm{~S} r \mathrm{NA}(\mathrm{H})$ was digoxigenin labelled and detected according to the manufacturer's protocol (Boehringer, Mannheim, Germany). Control experiments: the specificity of the cDNA probes was ascertained by computerised homology search and in situ hybridisation experiments in the fetal growth plate. ${ }^{21}$ Sense transcripts were used as non-specific negative controls and did not give signals above background (D).

Immunohistochemistry: deparaffinised sections were pretreated with testicular hyaluronidase and pronase. Primary antibodies were incubated for one hour and visualised using alkaline-phosphatase-labelled secondary antibodies. Nuclei were counterstained with haematoxylin. Polyclonal rabbit antisera against human type I collagen and monoclonal antibodies against type $X$ collagen were prepared as described elsewhere. ${ }^{27}{ }^{42}$ Monoclonal antibodies against type II collagen (CIID3) were kindly provided by Dr R Holmdahl (Uppsala, Sweden ${ }^{43}$ ). Polyclonal antibodies against type III procollagen were kindly provided by Dr Günzler (Frankfurt, Germany).

synthesising aggrecan, or merely reflects the fact that in the case of decreased proteoglycan synthesis the interterritorial proteoglycan content decreases first needs further investigation.

The hypoactivity of the upper osteoarthritic chondrocytes does not result from necrosis of the cartilage cells as demonstrated by the presence of cellular 18S rRNA (fig $1 \mathrm{H}$ ) and mRNA in these cells. ${ }^{1516}$ To understand the underlying mechanisms of the ongoing cellular events we have phenotyped the osteoarthritic chondrocytes using collagen gene expression as well established markers of chondrocyte differentia- tion in vivo and in vitro. ${ }^{19-22}$ Differentiated chondrocytes are known to express collagen types II, IX, XI, and aggrecan, while dedifferentiated chondrocytes express mainly collagen type I collagen besides some type III and V collagens, and hypertrophic chondrocytes are marked by the expression of collagen type X. Again, at the mRNA and protein level, we could demonstrate the expression of collagen type III (fig 2C, G) in the upper zone, where the chondrocytes switch from metabolically highly active cells to cells that no longer contribute to matrix balance. ${ }^{23}$ The uppermost 

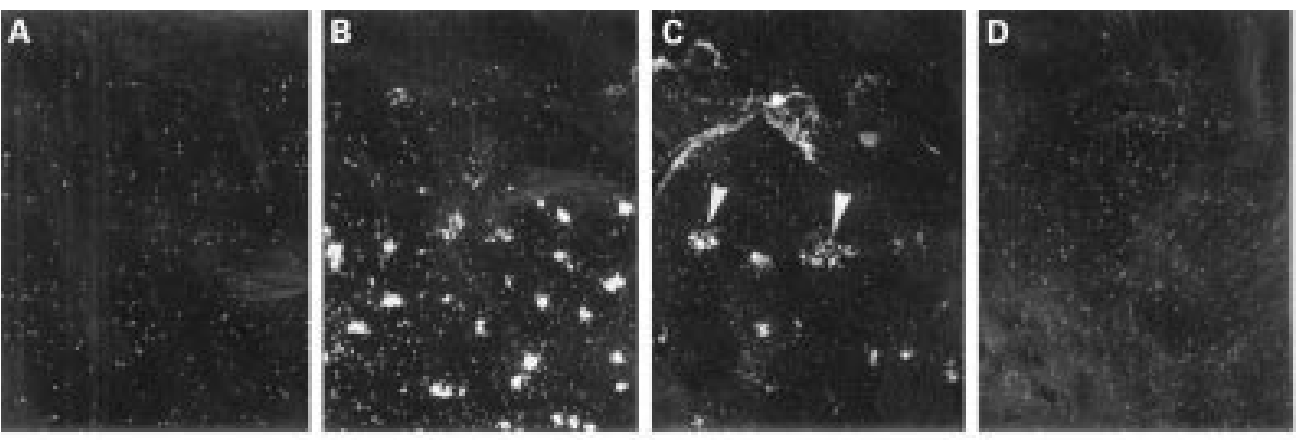

E
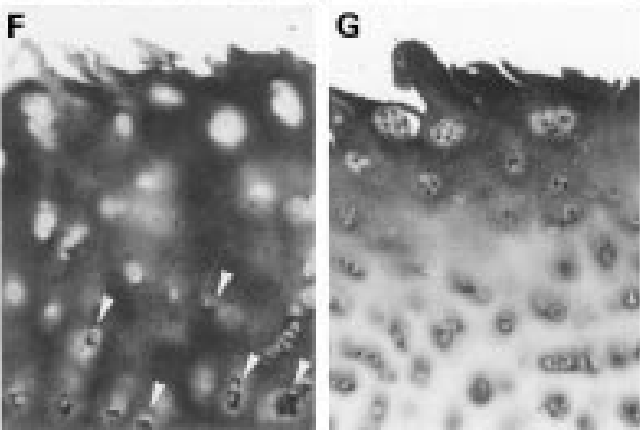

Figure 2 Phenotyping of osteoarthritic chondrocytes: in situ hybridisation analysis of chondrocytes revealed in all osteoarthritic specimens collagen type II $m R N A$ expression in the middle zones (B), where intracellular staining for type II collagen was also found immunohistochemically (F; arrow heads). In chondrocytes of the upper zone the expression of collagen type II ceased. Interestingly, in the upper middle zone an onset of type III collagen $m R N A$ expression was observed in most samples $(C)$. This was confirmed by immunohistochemical staining for collagen type III $(G)$. Neither collagen type $I(A, E)$ nor collagen type $X(D, H)$ expression or deposition, as marker collagens of dedifferentiated and hypertrophic chondrocytes, respectively, was observed in the upper and middle zones of osteoarthritic cartilage samples. Except for some weak signals for collagen type II, normal cartilage samples did not show expression of any of the collagens (not shown). ( $A$-D: dark fields; femoral head, 69 years, female; Mankin's grade 5; exposure time: A, C, D: three weeks; B: four days; original magnification $\times 100$ ).

chondrocytes often did not show the expression of collagen types I, II, III, or X (fig 2A-D). This differentiation pattern contrasts with the established modulations of the chondrocyte phenotype in vivo and in vitro, because so called 'dedifferentiated' chondrocytes express mainly collagen type $\mathrm{I}^{1924}$ and 'hypertrophic' chondrocytes express collagen type X..$^{2526}$ Neither of these were expressed by the osteoarthritic chondrocytes in the upper or middle zones in our specimens (fig $2 \mathrm{~A}, \mathrm{E}, \mathrm{D}, \mathrm{H}$ ). ${ }^{23}{ }^{27}$

\section{Hypothesis}

In situ analysis on the single cell level provides strong evidence that, although at first sight osteoarthritic cartilage degeneration seems to be a confusingly heterogeneous process, general rules can be established. Our hypothesis (fig 3), based on our studies and that of others, suggests a three step evolution of cellular events as one central feature during the osteoarthritic cartilage destruction process: (1) An increase of collagen type II and aggrecan synthesis. (2) Modulation of the chondrocytic phenotype with the expression of atypical gene products such as collagen type III. (3) Suppression of aggrecan core protein and collagen type II (and III) mRNA expression with subsequent quantitative loss of aggrecan molecules from the extracellular matrix. This results in an increase in the stress applied to the collagen network, ${ }^{28}$ thus promoting its destruction and thereby further loss of proteoglycans. $^{28}$ Physical damage to the collagen network occurs leading to fissuring and complete destruction of the cartilage matrix and cells. This process may begin in the superficial zone and progresses to the middle and deeper zones. Thus, different layers of osteoarthritic specimens represent different steps within this dynamic process.

Table 1 Typical results for normal and moderately damaged osteoarthritic cartilage (Mankin's grade 4-6). col: collagen; signal intensity: +++: very strong; ++: strong; +: weak; -: none; matrix integrity (histological): n: normal; f: fibrillated; : eroded). The different zones (surface, upper/lower middle, deep) are distinguished by criteria such as cell shape, matrix appearance, and distance to the subchondral bone and the tide mark

\begin{tabular}{|c|c|c|c|c|c|c|c|c|}
\hline \multirow[b]{2}{*}{$\begin{array}{l}\text { Cartilage } \\
\text { layer }\end{array}$} & \multicolumn{4}{|c|}{ Normal cartilage (Mankin's grade 0-2) } & \multicolumn{4}{|c|}{ Osteoarthritic cartilage (Mankin's grade 4-6) } \\
\hline & $\begin{array}{l}\text { Matrix } \\
\text { integrity }\end{array}$ & $\begin{array}{l}P G \\
\text { content }\end{array}$ & $\begin{array}{l}\text { Aggrecan } \\
\text { expression }\end{array}$ & $\begin{array}{l}\text { Phenotypic } \\
\text { markers }\end{array}$ & $\begin{array}{l}\text { Matrix } \\
\text { integrity }\end{array}$ & PG content & $\begin{array}{l}\text { Aggrecan } \\
\text { expression }\end{array}$ & $\begin{array}{l}\text { Phenotypic } \\
\text { markers }\end{array}$ \\
\hline Superficial & $\mathrm{n}$ & $-/+$ & $-/+$ & - & e & & & \\
\hline \multicolumn{9}{|l|}{ Middle } \\
\hline Upper & $\mathrm{n}$ & ++ & ++ & - & f & - & - & - \\
\hline Lower & $\mathrm{n}$ & ++ & ++ & - & (f) & ++ & +++ & -/col-III \\
\hline \multicolumn{9}{|l|}{ Deep } \\
\hline Upper & $\mathrm{n}$ & ++ & ++ & - & $\mathrm{n}$ & ++ & +++ & - \\
\hline Lower & $\mathrm{n}$ & ++ & ++ & - & $\mathrm{n}$ & ++ & +++ & col-X \\
\hline Calcified & $\mathrm{n}$ & + & $-/+$ & col-X & $\mathrm{n}$ & + & $-/+$ & col-X \\
\hline
\end{tabular}


Normal chondrocytes

low to moderate aggrecan synthesis
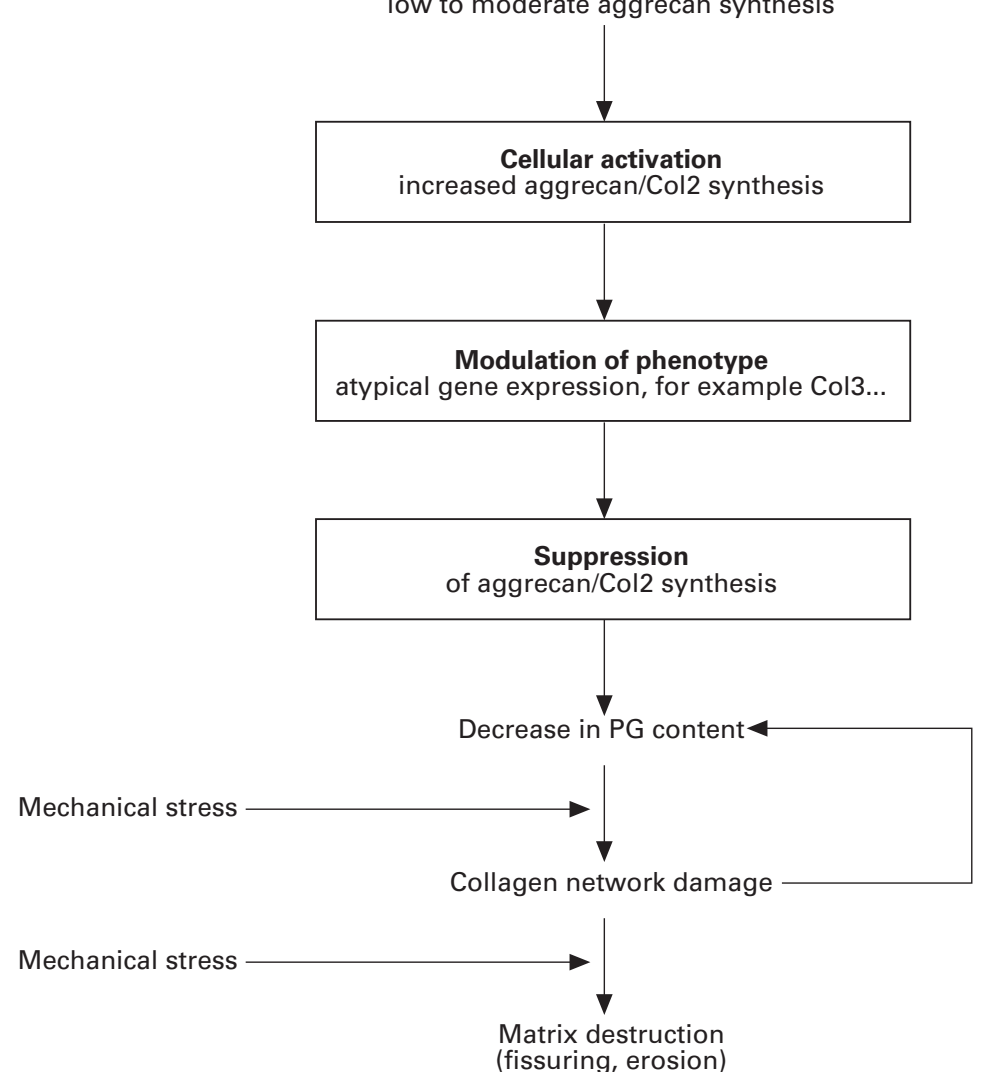

Figure 3 Schematic representation of the three steps of cellular events in osteoarthritic cartilage degeneration as proposed by the hypothesis: (1) cellular activation of chondrocytes, (2) modulation of the cellular phenotype, and (3) suppression of anabolic activity. This leads to a quantitative loss of aggrecan molecules from the extracellular matrix and to a collagen network damage, which again promotes further loss of proteoglycans. Finally, fissuring and complete destruction of the cartilage matrix occurs.

This concept does not preclude the importance of proteolytic degradation as a pathogenic mechanism within the osteoarthritic cartilage degeneration process or as a therapeutic target, but rather serves to focus this on the damage to the collagen network. ${ }^{8}$ Mature collagen fibres are large, multiple crosslinked rigid structures, which are presumably difficult to be repaired or replaced. Thus, preventing damage to pre-existing fibres is of primary importance. In contrast, aggrecan, having a faster turnover as demonstrated in normal articular cartilage, ${ }^{2930}$ can more easily be replaced by newly synthesised molecules. Thus, the inhibition of enzymatic activity, associated with proteoglycan turnover, might even hinder effective replacement of already damaged aggrecan molecules.

While the dynamic events described here may partly explain the osteoarthritic process, however, the factors that initiate this are not clear. These might be of a mechanical or an inflammatory nature leading to a limited damage, for example, to the collagen network. Most probably, several diverse initial events may lead to the same or a similar disease process, and the influence of cytokines such as interleukin 1 or tumour necrosis factor $\alpha$ may accelerate this. These mediators might act on a paracrine or autocrine level or diffuse into the articular cartilage from the synovial fluid.

\section{Testing of the hypothesis}

The data reported here and in other studies in support of the proposed hypothesis still need further confirmation as they have limitations, which are, however, at the moment difficult to resolve. ${ }^{1}$ Most studies, including ours, were done on peripheral areas of eroded tissue of late stage osteoarthritic material obtained at surgery. This always assumes that low to moderate Mankin's grades ${ }^{6}$ of late stages of osteoarthritic joints are comparable to cartilage of central areas of early or moderately advanced osteoarthritis. This, however, is not confirmed and needs further investigation, though initial studies with arthroscopic specimens seem to confirm results obtained in the late stage specimens. ${ }^{31}$ These studies have to be extended to include a larger number of specimens to prove the relevance of the process in the pathogenesis of osteoarthritis. ${ }^{2}$

In situ hybridisation as well as in situ metabolic labelling techniques do not provide ideal quantitative measurements. Nevertheless, the scoring system used represents an average score, and allows comparison of expression intensities, particularly within the same sample. ${ }^{3}$ It is also unresolved, why there are high interindividual differences in anabolic activity seen in all previous studies, whatever method applied. ${ }^{613}$ In this respect, the zonal distribution pattern described above is remarkably constant between specimens (in contrast with the absolute levels, which are quite different between the different samples).

Furthermore, it will be important to characterise further the 'osteoarthritic' cellular phenotype and to find additional markers to monitor the cellular processes.

\section{Future directions}

It will be an important goal in the future to establish in vitro systems reflecting these in vivo differentiation patterns of osteoarthritic chondrocytes to further analyse the disease process and to develop experimental systems to delay, stop, or even reverse it. This remains a possibility as chondrocytes, even in severely damaged areas, maintain the capacity to synthesise the necessary cartilage matrix components. ${ }^{29161832}$ Reversibility of switches in activity and differentiation have been demonstrated in vitro as well as in vivo in chondrocytes. ${ }^{33-35}$ Therefore, one can assume, that if further damage to the collagen network can be prevented, cartilage could repair itself or maintain the status quo because of the potentially high anabolic capacity of its cells. ${ }^{29}$

It has to be borne in mind, however, that re-activation of phenotypically modulated chondrocytes is of little help as it may only increase non-cartilaginous matrix gene expression at the expense of essential matrix components such as aggrecan. ${ }^{3637}$ This situation would be similar to the increase in non-cartilaginous protein synthesis of dedifferentiated chondrocytes stimulated by fetal calf serum. ${ }^{38}$ Instead, the stabilisation of the chondrocytic phenotype and redifferentiation of the osteoarthritic chondrocytes is needed to ensure correct matrix anabolism. Factors of the 
transforming growth factor $\beta$ superfamily, in particular bone morphogenetic proteins such as OP-1 (osteogenin) ${ }^{39} 40$ and BMP-2, ${ }^{44}$ might be suitable agents to initiate this.

This work was supported by the Deutsche Forschungsgemein schaft (DFG Grant Ai 20/1-1).

We thank Ms G Herbig for expert photographic and Ms M Mauser and Ms M Schaller for expert technical assistance.

1 Thompson RC, Oegema TR. Metabolic activity of articular cartilage in osteoarthritis. J Bone Joint Surg 1979;A59:40716.

2 Mankin HJ, Johnson ME, Lippiello L. Biochemical and metabolic abnormalities in articular cartilage from osteoarthritic human hips. J Bone Joint Surg 1981;63:131-9.

3 Sweet BME, Thonar EJMA, Immelman AR, Solomon L. Biochemical changes in progressive osteoarthrosis. Ann Biochemical changes in progr
Rheum Dis 1977;36:387-8.

4 Mendler M, Eich-Bender SG, Vaughan L, Winterhalter KH, Bruckner P. Cartilage contains mixed fibrils of colla$\mathrm{KH}$, Bruckner P. Cartilage contains mixed fibrils of
gen types II, IX and XI. J Cell Biol 1989;108:191-7.

5 Roughley P J, Lee E R. Cartilage proteoglycans: structure and potential functions. Microsc Res Techn 1994;28:38597

6 Mankin HJ, Dorfman H, Lippiello L, Zarins A. Biochemical and metabolic abnormalities in articular cartilage from osteo-arthritic human hips. J Bone Joint Surg 1971; 53A:523-37.

7 Poole AR. Changes in the collagen and proteoglycan of articular cartilage in arthritis. Rheumatology 1986; 10:316-71

8 Dodge GR, Poole AR. Immunohistochemical detection and immunohistochemical analysis of type II collagen degradation in human normal, rheumatoid, and osteoarthritic articular cartilages and in explants of bovine articular cartilage cultured with interleukin 1. J Clin Invest 1989; tilage culture

9 Rizkalla G, Reiner A, Bogoch E, Poole AR. Studies of the articular cartilage proteoglycan aggrecan in health and articular cartilage proteoglycan aggrecan in
osteoarthritis. J Clin Invest 1992;90:2268-77.

10 Lippiello L, Hall MD, Mankin HJ. Collagen synthesis in normal and osteoarthrotic human cartilage. J Clin Invest 1977;59:593-600.

11 Nimni ME, Deshmukh K. Differences in collagen metabolism between normal and osteoarthritic human articular cartilage. Science 1973;181:751-2

12 Eyre DR, McDevitt CA, Billingham ME J, Muir H. Biosynthesis of collagen and other matrix proteins by articular cartilage in experimental osteoarthrosis. Biochem J 1980;188:823-37.

13 Sandy JD, Adams ME, Billingham MEJ, Plaas A, Muir H. In vivo and in vitro stimulation of chondrocyte biosynthetic activity in early experimental osteoarthritis. Arthritis Rheum 1984;27:388-97.

14 Matyas JR, Adams ME, Huang D, Sandell LJ. Discoordinate gene expression of aggrecan and type II collagen in
experimental osteoarthritis. Arthritis Rheum 1995; 38:420-5.

15 Aigner T, Stö $\beta$ H, Weseloh G, Zeiler G, von der Mark K Activation of collagen type II expression in osteoarthritic and rheumatoid cartilage. Virchows Arch B 1992;62:33745 .

16 Aigner T, Vornehm SI, Zeiler G, Dudhia J, von der Mark K, Bayliss MT. Suppression of cartilage matrix gene expression in upper zone chondrocytes of osteoarthritic chondrocytes. Arthritis Rheum (in press)

17 Lafeber FPJG, van der Kraan PM, van Roy JLAM, van den Berg WB, Bijlsma JWJ. Local changes in proteoglycan synthesis during culture are different for normal an

18 Grushko G, Schneiderman R, Maroudas A. Some biochemical and biophysical parameters for the study of the pathogenesis of osteoarthritis: a comparison between the processes of aging and degeneration in human hip carthe processes of aging and degeneration in hum
tilage. Connect Tissue Res 1989;19:149-76.

19 von der Mark K, Gauss V, von der Mark H, Müller PK. Relationship between cell shape and type of collagen synRelationship between cell shape and type of collagen syn-
thesized as chondrocytes lose their cartilage phenotype in thesized as chondrocytes lose their

20 Cancedda R, Descalzi-Cancedda F, Castagnola P. Chondrocyte differentiation. Int Rev Cytol 1995;159:265-358.

21 Vornehm SI, Dudhia J, von der Mark K, Aigner T. Expression of collagen types IX and XI as well as other major cartilage matrix components by human fetal chondrocytes in vivo. Matrix Biol 1996;15:91-8.
22 Aigner T, Dietz U, Stöß H, von der Mark K. Differential expression of collagen types I, II, III, and $\mathrm{X}$ in human expression of collagen types I, II, III, a
osteophytes. Lab Invest 1995;73:236-43.

23 Aigner T, Bertling W, Stö $\beta$ H, Weseloh G, von der Mark K. Independent expression of fibril-forming collagens I, II, and III in chondrocytes in human osteoarthritic cartilage. J Clin Invest 1993;91:829-37.

24 Benya PD, Padilla SR, Nimni ME. Independent regulation of collagen types by chondrocytes during the loss of differentiated function in culture. Cell 1978;15:1313-21.

25 Reichenberger E, Aigner T, von der Mark K, Stö $\beta$ H, Bertling W. In situ hybridization studies on the expression of type X collag

26 Schmid TM, Popp RG, Linsenmayer TF. Hypertrophic cartilage matrix. Ann N Y Acad Sci 1990;580:64-73.

27 Girkontaité I, Frischholz S, Lammi P, Wagner K, Swoboda $\mathrm{B}$, Aigner $\mathrm{T}$, et al. Immunolocalization of type X collagen in normal fetal and adult osteoarthritic cartilage with monoclonal antibodies. Matrix Biol 1996;15:231-8.

28 Maroudas A. Balance between swelling pressure and collagen tension in normal and degenerate cartilage. Nature 1976;260:808-9.

29 Hardingham TE, Fosang AJ, Dudhia J. Aggrecan, the chondroitin sulfate/keratan sulfate proteoglycan from cartilage. Kuettner K, Schleyerbach R, Peyron JG, eds. Articular cartilage and osteoarthritis. New York: Raven Press, 1992:5-19.

30 van Kampen GPJ, van de Stadt RJ, van de Laar AFJ, van der Korst JK. Two distinct metabolic pools of proteoglycans in articular cartilage. Kuettner K, Schleyerbach R, Peyron JG, Hascall V, eds. Articular cartilage and osteoarthritis. New York: Raven Press, 1992: 281-9.

31 Aigner T, Glückert K, von der Mark K. Activation of fibrillar collagen synthesis and phenotypic modulation of chondrocytes in early human osteoarthritic cartilage lesions. Osteoarthritis Cartilage (in press).

32 Mitrovic D, Gruson M, Demignon J, Mercier P, Aprile F, de Seze S. Metabolism of human femoral head cartilage in osteoarthrosis and subcapital fracture. Ann Rheum Dis $1981 ; 40: 18-26$.

33 Benya PD, Shaffer JD. Dedifferentiated chondrocytes reexpress the differentiated collagen phenotype when cultured in agarose gels. Cell 1982;30:215-24.

34 Rayan V, Hardingham TE. The recovery of articular cartilage in explant culture from interleukin-1a: effects on proteoglycan synthesis and degradation. Matrix Biol proteoglycan synth

35 Bonaventure J, Khadom $\mathrm{N}$, Cohen-Solal L, $\mathrm{Ng} \mathrm{KH}$, Bourguignon J, Lasselin C, et al. Re-expression of cartilage-specific genes by dedifferentiated human articular chondrocytes cultured in alginate beads. Exp Cell Res 1994;212:97-104

36 Little CB, Ghosh P, Bellenger CR. Topographic variation in biglycan and decorin synthesis by articular cartilage in the early stages of osteoarthritis: an experimental study in sheep. J Orthop Res 1996;14:433-44.

37 Doré S, Pelletier J-P, Dibattista JA, Tardif G, Brazeau P, Martel-Pelletier J. Human osteoarthritic chondrocytes possess an increased number of insulin-like growth factor possess an increased number of insulin-like growth factor
1 binding sites but are unresponsive to its stimulation. Arthritis Rheum 1994;37:253-63.

38 Kato Y, Gospodarowicz D. Growth requirements of low-density rabbit costal chondrocyte cultures maintained in serum-free medium. J Cell Physiol 1984;120:354-63.

39 Flechtenmacher J, Huch K, Thonar EJ-MA, Mollenhauer J, Davies SR, Schmid T, et al. Recombinant human osteogenic protein 1 is a potent stimulator of the synthesis of cartilage proteoglycans and collagens by human articular chondrocytes. Arthritis Rheum 1996;39:1896-904.

40 Harrison ET, Luyten FP, Reddi AH. Osteogenin promotes reexpression of cartilage phenotype by dedifferentiated articular chondrocytes in serum-free medium. Exp Cell Res 1991;192:340-5.

41 Rosenberg LC. Chemical basis for the histological use of safranin $\mathrm{O}$ in the study of articular cartilage. J Bone Joint Surg 1971;53A:69-82

42 von der Mark K, Kirsch T, Nerlich AG, Kub A, Weseloh G, Glückert $\mathrm{K}$, et al. Type X collagen synthesis in human osteoarthritic cartilage. Arthritis Rheum 1992;35:806-11.

43 von der Mark K, von der Mark H, Gay SW. Study of differential collagen synthesis during development of the chick embryo by immunofluorescence. Dev Biol 1976;53:15370.

44 Sailor LZ, Hewick RM, Morris EA. Recombinant human bone morphogenetic protein-2 maintains the articular chondrocyte phenotype in long-term culture. J Orthop Res 1996;14:937-45. 